\title{
Pregnancy of un-known location: need for evolving new protocols and evaluation of tools for increasing outcome predictability
}

\begin{abstract}
Aim: To find out the timeline for diagnosis and outcome of pregnancy of un-known location PUL cases. To find out the approach for accuracy of diagnosis and to prevent doing unnecessary scan follow ups and $\beta \mathrm{HCG}$ tests.

Method: Prospective observational study over one year period between January 2015 and January 2016.

Result: In this study 50patients were included and six patients were excluded as they didn't meet the criteria. The Intra-uterine Pregnancy (IUP) was diagnosed in $28 \%(n=14)$. The suboptimal $\beta H C G$ was seen in $11 \%(n=5)$ patients, who later were diagnosed with ectopic pregnancy. The remaining 56\% $(n=25 / 44)$ patients unfortunately had failing IUP, and none of them were labelled as persistent PUL. The number of $\beta$ HCG tests undertaken in Ectopic group ranged from 3-8, compared to 2-6 times in failing IUP group whereas in IUP cohort it remained one to two tests. Similarly, we found that number of pelvic scan required for making diagnosis in three above-mentioned groups varied. Our study data revealed that each patient had 2-4 scans in Ectopic group, and 1-3 scans needed in Failing IUP.
\end{abstract}

Volume 7 Issue I - 2018

\author{
Junaid Rafi,' Haroona Khalil, ${ }^{2}$ Geetha \\ Mahindrakar ${ }^{3}$ \\ 'Registrar Obstetric \& Gynecology, Ipswich Hospital NHS Trust, \\ United Kingdom \\ ${ }^{2}$ Consultant Obstetric \& Gynecology, lpswich Hospital NHS \\ Trust, United Kingdom \\ ${ }^{3}$ Consultant Obstetric \& Gynecology, Ysbyty Gwynedd, United \\ Kingdom
}

Correspondence: Junaid Rafi, MBBS, MRCPI, DFSRH, Registrar Obstetric \& Gynecology, Ipswich Hospital NHS Trust, Heath Road, IP4 5PD, United Kingdom, Tel 0044-77809|8558, Email drjunaidrafi@hotmail.com

Received: December 28, 2017 | Published: January 05, 2018
Conclusion: To reach the final definite diagnosis / outcome in PUL cases in set time limit may not be possible in some atypical presentation of early pregnancy problems especially when the aim is to avoid un-necessary scans, follow ups and blood test without compromising the safety. Therefore we suggested a new local protocol for diagnosing and managing atypical presentations aiming to achieve outcome in 5 to 9days durations.

Keywords: pregnancy un-known location, PUL, ectopic pregnancy

\section{Background}

Ascertaining pregnancy location is the key determinant for streamlining management for patients presenting with abdominal pain or bleeding in early pregnancy. However sometimes in spite of all efforts finding the location of gestation sac is difficult and such cases have been classified categorised as Pregnancy of Unknown Location (PUL). The lack of well accepted consensus for diagnostic criteria for PUL is reflected by differences in the management strategies of PUL. As locating early pregnancy is dependent on many factors therefore incidence of PUL varies in different setup. According to recent reports the Specialist unit reported that to around $8-10 \%$ vs $8-31 \%$ in non-specialist Unit. ${ }^{1}$ PUL outcome data is very encouraging from hospitals that have developed clear PUL care pathways incorporating reliable diagnostic criteria. Only few UK units have opted their PUL management based on a clear predictive tool including widely accepted hormone tests. Hence we conducted a prospective study to review PUL managed cases and aimed to propose a PUL management pathway and thereby reducing variation.

\section{Aim}

The aim was to find out the timeline for diagnosis and outcome of PUL cases and to propose the prompt and effective approach for PUL diagnosis. Also wanted to find out the pattern and frequency of scans and BHCG in PUL cases.

\section{Criteria}

PUL cases were identified if there was no evidence of intrauterine or extra uterine pregnancy on first transvaginal ultrasound scan (56 week gestation) in women with a positive pregnancy test.

\section{Methods}

This was a prospective observational study, including all patients attending EGAU (Early Gynaecology Assessment Unit) who were diagnosed as PUL between January 2015 and January 2016 at District General Hospital, and cases were followed up for object analysis of outcome. The proforma for prospective data collection was started by the nurses and all information of consultation was recorded. All proformas were collectively analysed at the end of study.

\section{Result}

Fifty patients who attended EGAU were included in this PUL study. Six patients were excluded as they didn't meet the criteria of PUL study protocol. The $28 \%(\mathrm{n}=14)$ found to have intra-uterine pregnancy (IUP). The suboptimal $\beta$ HCG rise was seen in $11 \%(n=5)$ patients, who later were diagnosed with ectopic pregnancy. The remaining 56\% $(\mathrm{n}=25 / 44)$ patients failing IUP and none of them were labelled as persistent PUL (Figure 1). The serum $\beta \mathrm{HCG}$ assessments were undertaken 3-8times before reaching diagnosis in Ectopic group 
whereas in Failing IUP group serum $\beta$ HCG was done 2-6times and in IUP cohort it remained one to two tests. Our data revealed that number of scans required for each patient in above mentioned three groups varied. For ectopic group patients had 2-4 scans, and patients in failing IUP \& IUP group required an average 1-3 pelvic scans (Table 1). In cases which required more than three $\beta H C G$ the management was discussed with consultant on call for further follow up. The analysis of ectopic pregnancy group revealed that suboptimal rise in serum $\beta H C G$ and ultrasound scan findings were the main factors considered leading to the diagnosis of ectopic. All patients in ectopic group remained hemodynamically stable. The mean time to establish diagnosis in ectopic group was 6-21days, and serum $\beta H C G$ discriminatory zone values ranged (1500- $2400 \mathrm{IU} / \mathrm{L})$. One case diagnosed as ectopic pregnancy on $21^{\text {st }}$ day of reporting EGAU, while the serum $\beta H C G$ values were $<1500 \mathrm{IU} / \mathrm{L}$ and patient had Laparoscopic salpingectomy. The other such case managed with Methotrexate where $\beta$ HCG was $<500$ IU/L

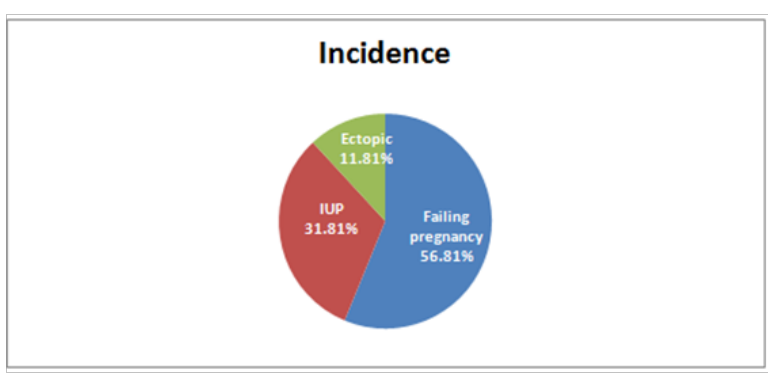

Figure I Outcome PUL cases.

Table I Frequency of $\beta \mathrm{HCG}$ and Transvaginal ultrasound scans in each patient

\begin{tabular}{llll}
\hline $\begin{array}{l}\text { Outcome } \\
\text { group }\end{array}$ & $\begin{array}{l}\text { No. Scans } \\
\text { per patient }\end{array}$ & $\begin{array}{l}\text { No. of } \boldsymbol{\beta H C G} \\
\text { per patient }\end{array}$ & $\begin{array}{l}\text { Mean time of } \\
\text { diagnosis established } \\
\text { (Days) }\end{array}$ \\
\hline $\begin{array}{l}\text { Failing } \\
\text { Pregnancy }\end{array}$ & $1-3$ & $2-6$ & $2-32$ \\
Ectopic & $2-4$ & $3-8$ & $6-21$ \\
& $1-2$ & $1-2$ & $2-14$ \\
\hline IUP & & & \\
\hline
\end{tabular}

\section{Discussion}

The term 'Pregnancy of Unknown Location' (PUL) is referred when there is no conclusive evidence of either intra- or extra uterine pregnancy or retained products of conception on transvaginal ultrasound, despite a positive pregnancy test. ${ }^{2}$ The issue with PUL are as below

i. Overtreatment in the form of early laparoscopy since there is false negative rate of $3-4 \%$ when undertaken done too early and $5 \%$ false positive rate of laparoscopy are due to the presence of retrograde uterine bleeding in miscarriage which can be misinterpreted as tubal ectopic pregnancy.

ii. Fear of missing Ectopic pregnancy with "Wait \& see conservative policy".

iii. No clear defined role of methotrexate in PUL cases and concern of using in early pregnancy of uncertain viability.

iv. Multiple follow ups with Blood (BHCG, Progesterone) investigation with not well-established predictive value.

v. Repeated ultrasound scans follow up and issues with reporting and expertise in specialised versus non specialised units.

Less consistency in scan report quality in non-specialist unit has been recognised as an issue. This trend was seen in our study as well requiring up to four scan in some patients of ectopic group and three scans in failing IUP group. As there are still no conclusive RCT (randomised controlled trials) published to compare different management strategies for PUL. There seemed a clinical heterogeneity between the studied populations; highlights to revisit the policy to adhere to specific models extrapolated from studies conducted in relatively bigger specialist units where possible best expertise and equipment are available. It is rather advisable to develop management PUL protocol locally; taking into the consideration demographics of population and available facilities and expertise. We proposed a new protocol shown below based on findings from our study (Figure 2). This pathway B \& C (Figure 2) follows the same flow as per NICE Assessment of pregnancy of un known location interactive flow chart $^{3}$ but differs from point $\mathrm{A}$ to further into $\mathrm{A} 1, \mathrm{~A} 2$ and $\mathrm{A} 3$. It can be argued that setting a target (time limit) to reach definitive diagnosis may not be achievable in all patients but in new proposed protocol total duration from initial presentation to final treatment offered will be 9,7 and 5days in Pathway A.1,A.2 and A.3 respectively (Figure 2 ) on contrary to initial finding of diagnosis of 2-32days for failing pregnancy and ranged between 6-21days for ectopic pregnancy.

There are number of different models designed to define and classify PUL management based on various mathematical calculations, including $\beta H C G$ with or without progesterone levels. Two protocols seemed more pragmatic to implement safely in our unit for the prediction of failing group and early intrauterine pregnancy group.

\section{Model I}

One was proposed by Condous et al., ${ }^{4}$ that the serum $\beta \mathrm{HCG}$ ratio at $48 \mathrm{hr}$ : $0 \mathrm{hr}<0.87$ promises an optimal test for the prediction of failing pregnancy. It was concluded from our study that serum $\beta \mathrm{HCG}$ ratio $<0.87$ (Table 2) can easily and safely be used for predicting failing pregnancy in PUL cases $(>50 \%)$

\section{Model 2}

The other model was suggested by Bignardi et al., ${ }^{5}$ who concluded that $\beta \mathrm{HCG}$ ratio $48 \mathrm{hr}: 0 \mathrm{hr}>2.00$ points toward increased chances for a viable IUP and our study proved that serum $\beta$ HCG ratio $>2.0$ (Table 3 ) can be helpful in predicting ongoing early intrauterine pregnancy in PUL cases $(>60 \%)$. 
Table 2 Validity of $\beta \mathrm{HCG}$ level ratio @ $48 \mathrm{~h} / 0<0.87$ as predictive tool for failing pregnancy

\begin{tabular}{ll}
\hline BHCG 48hr/0 hr ratio & $\begin{array}{l}\text { Number of cases out of total failing pregnancy group } \\
(\mathbf{n} / \mathbf{2 5})\end{array}$ \\
\hline $\begin{array}{l}\text { Number of cases (n) in failing pregnancy group where } \beta \text { HCG ratio was } \\
<0.87\end{array}$ & $14 / 25(56 \%)$ \\
$\begin{array}{l}\text { Number of cases (n) in failing pregnancy group where } \beta \text { HCG ratio was } \\
>0.87\end{array}$ & $10 / 25(40 \%)$
\end{tabular}

Table 3 Validity of $\beta$ HCG level ratio @ 48h/0h >2.00 as predictive tool for a viable Intrauterine pregnancy (IUP)

\begin{tabular}{lc}
\hline $\boldsymbol{\beta H C G} 4 \mathbf{4 h r} / \mathbf{0}$ hr ratio & Number of cases out of total IUP group (n/25) \\
\hline Number of cases (n) in early IUP group where $\beta$ HCG ratio was $>2$ & $9 / 14(64 \%)$ \\
Number of cases (n) in early IUP group where $\beta$ HCG ratio was $>1.6-2$ & $5 / 14(35 \%)$
\end{tabular}

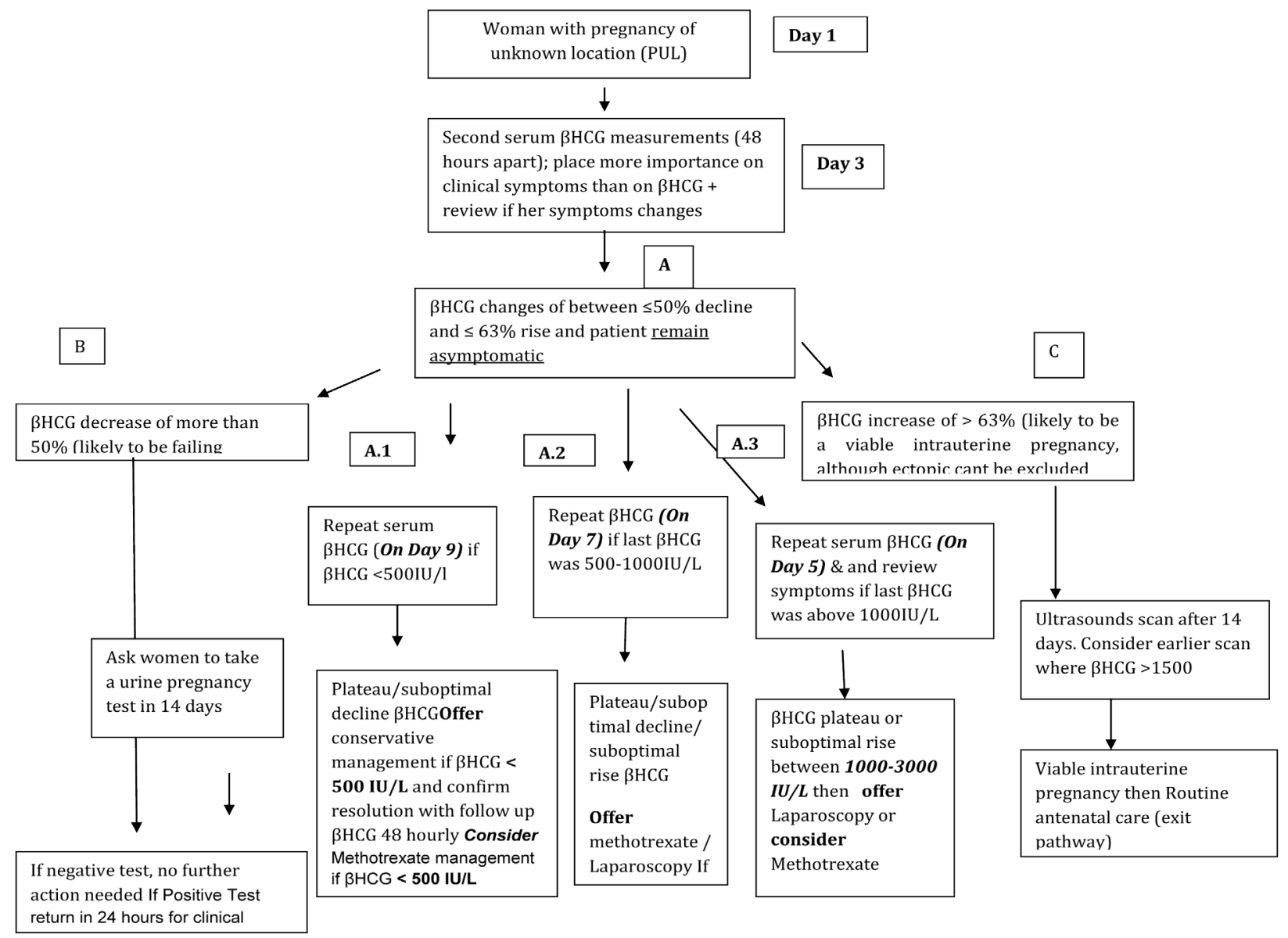

Pathway

i. A.I: for Plateau or suboptimal decline $\beta \mathrm{HCG}$ values $<500 \mathrm{IU} / \mathrm{L}$ : Total duration from Initial presentation (Day I) to treatment offered comprise of 9 days

ii. Pathway A.2: for Plateau or suboptimal decline $\beta \mathrm{HCG}$ values $500 \mathrm{IU} / \mathrm{L}-\mathrm{I000IU/L}$ : Total duration from Initial presentation (Day I) to treatment offered comprise of 7 days

iii. Pathway A.3: for Plateau $\beta$ HCG values of $>1000 \mathrm{IU} / \mathrm{L}$ and above:Total duration from Initial presentation (Day I) to treatment offered comprise of 5 day.

Figure: 2 Pregnancy Of Unknown location Management Protocol. 


\section{Strengths and limitations}

This was a small but prospective study conducted in District General Hospital but a sample size of more than 40 was taken to ensure that the sample mean is approximately normally distributed. All proformas were collectively analysed at the end of study to reduce the risk of bias and influence on management during the study period. The inferences drawn from this study may not be applicable nationally for National guidelines but hopefully smaller early pregnancy assessment units may benefit from finding of our study in developing and testing new departmental protocols.

\section{Conclusion}

To reach the final definite diagnosis/outcome in PUL cases in set time limit may not be possible in some atypical presentation of early pregnancy problems especially when the aim is to avoid un-necessary scans, follow ups and blood test without compromising the safety. Therefore we suggested a protocol for diagnosing and managing atypical presentations of PUL.

\section{Recommendation}

i. Introduction of local pathways where initial presentation to final treatment will be offered 9days (serum $\beta \mathrm{HCG}<500 \mathrm{IU} / \mathrm{L}$ group), 7 days (serum $\beta$ HCG 500-1000IU/L group) and 5days where BHCG will be $>1000 \mathrm{IU} / \mathrm{L}$. It may help reducing unnecessary follow ups and may streamline management of difficult scenarios of plateauing serum $\beta$ HCG.

ii. Audit in a year's time to review the results to ensure safety.

iii. Use of methotrexate in selective hemodynamically stable patients as per NICE guideline ${ }^{6}$ after counselling and careful consideration that possibility of early viable pregnancy is ruled out.

\section{Authors contribution}

i. JR designed and conducted the study and did manuscript writing.

ii. HK did editing manuscript, review and final approval of manuscript.

iii. GM Collected data with JR and helped writing manuscript.

\section{Acknowledgements}

None.

\section{Conflict of interest}

The author declares no conflict of interest.

\section{References}

1. Sagili H, Mohamed K. Pregnancy of unknown location: an evidencebased approach to management. The Obstetrician \& Gynaecologist. 2008;10:224-230.

2. Royal college of obstetricians and gynaecologists. The Management of Early Pregnancy Loss. USA: Green- top; 2006.

3. NICE Assessment of pregnancy of un known location interactive flow chart.

4. Condous G, Van Calster B, Kirk E, et al. Prospective cross-validation of three methods of predicting failing pregnancies of unknown location. Hum Reprod. 2007;22(4):1156-1160.

5. Bignardi T, Condous G, Alhamdan D, et al. The HCG ratio can predict the ultimate viability of the intrauterine pregnancies of uncertain viability in the pregnancy of unknown location population. Hum Reprod. 2008;23(9):1964-1967.

6. Ectopic Pregnancy and miscarriage: Diagnosis and initial management in early pregnancy of ectopic pregnancy and miscarriage (CG154). USA: NICE; 2012. p. 1-39. 